\title{
The Questionnaire Survey on Computer Basis in 2016 Freshmen and The Graded Teaching Suggestions

\author{
Xueyun Wang ${ }^{1}$, Xuelian $\mathrm{Hou}^{2}$ and Xiaorong Zhang ${ }^{1}$
}

${ }^{1}$ Shandong College of Electric Power Department of Information Engineering, Shandong Taian 271000; 2 State Grid of China Technology College Shandong Taian 271000

\begin{abstract}
As for the large gap of computer level among 2016 freshmen, the questionnaire survey based on Computer Application Basis is used to investigate the 2016 freshmen. Through the statistical analysis of the survey's results , about $50 \%$ of the freshmen's computer foundation is poor, about $40 \%$ of the students have a certain degree of computer operation ability and $10 \%$ of the students have a higher computer application abilities. According to the investigation results, this paper puts forward some suggestions on the graded teaching and discusses the concrete implementation process and the hierarchical teaching method.
\end{abstract}

Keywords: Freshman; Computer base; Survey; Questionnaire; Grading teaching

\section{6 级新生计算机基础问卷调查及分级教学建议}

\author{
王雪笏 $1^{1}$, 侯雪莲 $2^{2}$, 张晓蓉 $1^{1}$ \\ （1. 山东电力高等专科学校信息工程系，山东省 泰安市 271000 ;
}

2. 国网技术学院 山东省 泰安市 271000)

摘要: 针对 2016 级新生的计算机基础水平差距过大的问题，参考《计算机应用基础》课程教学内容设计调查问卷，对 2016 级新生进行问卷调查。通过对调查结果的统计分析，可知约 50\%的新生计算机基础较差，约 40\%的学生有一定的计算 机操作能力, 有 $10 \%$ 的新生有较高的计算机应用能力。根据调查结果, 提出了分级教学的建议, 并论述了具体实施过程和 分级教学方法。

关键词: 新生; 计算机基础; 调查; 问卷; 分级教学

中图分类号：TP391.1 文献标志码：A

引言

《计算机应用基础》是我校开设的一门基本素质和通用能力课程, 适用于所有专业。本课程的教学目 标是通过学习, 使学生能够掌握计算机的基本知识和技能; 熟悉 Windows 操作系统和 Office 办公软件的 基本操作; 了解计算机网络、多媒体技术及信息安全的基本概念; 学会利用所学知识和技能解决实际问题, 提高计算机文化素养, 适应未来工作的需要, 并为后续的计算机课程学习打下良好的基础。

这门课程与其他课程在教学上的不同之处是学生在入学前已有了一定的计算机基础, 但是差别很大。 按照教育部要求, 从 2001 年起, 全国中小学开设信息技术课程, 但是因为各地区对信息课程的重视程度 不同、各个学校的计算机教学硬件及师资的差别, 使得每年入学新生的计算机应用能力有相当大的差距。 这就给计算机教学造成了很大的困扰。面对一个知识点, 授课教师认为需要详细讲解, 可有的同学早已熟 练掌握, 甚至比老师懂得还多, 因此课堂上就不愿意集中精力听讲, 说话、玩手机, 甚至扰乱课堂纪律; 有些简单操作, 授课教师觉得同学们应该都掌握了, 只需简单介绍即可, 没想到个别学生对这些最基本的 操作一埪不通, 甚至从来没有接触过计算机, 必须从零开始学习。对教师来说, 迁就哪一方都不好, 只好 采取折中的方式, 针对大部分也就是有一定基础, 但又不十分熟练的学生来组织教学。容易导致有的学生 
“吃不饱”，有的学生“吃不了”。

\section{1 调查问卷内容设计}

为了对新生的计算机水平有一个具体的了解, 我参照课程教学内容设计了一份调查问卷, 选取了 2016 级 5 个新生班级进行问卷调查。调查问卷的问题设计既有封闭式单选或多项选择题，也有开放式的补充回 答。主要内容包括:

(1) 学生上中学时是否有信息课及信息课里学到哪些知识;

（2）家里是否有计算机，如果有计算机，主要用来干什么? 在这里列出 5 个选项: 上网汶览、聊天、 玩游戏、学习、查资料, 学生可以选择其中的几项, 也可以自己补充。

（3）针对本课程要学的 office 办公软件Word、Exce1、Powerpoint、Frontpage 及 Inernet 应用, 各自选出 4 个或 5 个有代表性的操作让学生选出会做的选项, 如 Word 有文档编辑、文档排版、插入表格、 插入图片 4 个选项，一共是 21 个可选操作。学生还可补充其他会做的操作。

（4）针对这门课需要掌握的计算机网络、多媒体技术、信息安全等基础知识, 选出了有代表性的 5 个知识点：计算机网络拓扑结构、DNS 域名系统、声音采样频率、防火墙的概念、计算机病毒的防治, 让 学生来选择或补充。

(5) 调查新生了解或掌握哪种编程语言及掌握程度（一般或熟练）。

\section{2 调查过程}

调查时机为新生入学一个月以内, 利用各班级在机房上课时间进行调查, 一般是下课前 15 分钟。首 先说明此项调查的目的: 了解同学们的计算机水平, 以便教学时能有的放矢; 同时强调要求同学们根据自 己的实际情况填写调查问卷, 以保证调查结果的客观公正。然后通过广播教学系统下发电子问卷, 学生填 写保存后匿名提交。

\section{3 调查结果}

通过对调查问卷的回收、整理和统计，得到如下结果：

\section{1 根据每个选项得出的统计结果}

(1) 大约有 $88 \%$ 的学生在中学期间开设有信息课。在这部分学生中, 有 55\%选择学过文字处理, 有 $61 \%$ 的学生学过表格制作, 41\%的学过制作幻灯片, 25\%的学生学过网页制作, 57\%会上网汶览。还有个别同学 补充学过图像处理和动画制作。

(2) 大约有 70\%的学生家里有计算机。对于计算机的用途, 在这部分学生中, 选择学习的只占 $40 \%$, 选择查资料的占 $74 \%$ ，选择娱乐项目的占绝大多数，如上网汶览 $94 \%$ ，聊天 $91 \%$ ，玩游戏 $80 \%$ 。

(3) office 办公软件Word、Excel、Powerpoint、Frontpage 及 Inernet 应用的掌握情况如表 1 所示。

表 1

\begin{tabular}{c|c|c|c|c|c|c}
\hline 软件 & 操作 1 & 操作 2 & 操作 3 & 操作 4 & 操作 5 & 平均值 \\
\hline Word & $25 \%$ & $30 \%$ & $52 \%$ & $56 \%$ & $40.8 \%$ \\
\hline Excel & $42 \%$ & $24 \%$ & $40 \%$ & $26 \%$ & $24 \%$ & $31.2 \%$ \\
\hline Powerpoint & $44 \%$ & $26 \%$ & $26 \%$ & $28 \%$ & $31 \%$ & $25.5 \%$ \\
\hline Frontpage & $22 \%$ & $32 \%$ & $44 \%$ & $4 \%$ & & $62 \%$ \\
\hline Inernet 应用 & $22 \%$ & $64 \%$ & $86 \%$ & $76 \%$ & & $2 \%$ \\
\hline
\end{tabular}


（4）有关计算机网络、多媒体技术、信息安全等的 5 个知识点，分别有 $8 \% 、 20 \% 、 12 \% 、 26 \% 、 26 \%$ 的学生选择了解, 平均 $18.4 \%$;

(5) 学生在中学时接触过的计算机编程语言, 其中学过 $\mathrm{C} / \mathrm{C}++$ 的最多, 占 $3.5 \%$, 其它个别学生学过 VB、PLC、Matlab 等, 掌握程度都是一般了解。

\section{2 根据每份调查问卷的 21 个操作和 5 个知识点得出的统计结果}

26 个操作或知识点中, 最少的选择只会 1 个, 最多的选择会 22 个。分 3 个区间进行统计：其中选择 会 1 8 个的占 52\%, 会 9 16 个的占 38\%, 会 17 26 个的占 $10 \%$ 。分 2 个区间进行统计: 其中选择会 1 13 个的占 $72 \%$, 会 $14 \sim 26$ 个的占 $28 \%$ 。

\section{4 调查结果分析}

根据以上调查结果可知:

（1）绝大部分同学在中学时上过信息课, 学过计算机基本操作, 但是因为不是考试科目, 没有引起 足够的重视, 基础并不牢固。有的同学在填写调查问卷时犹豫不定, 因为不少知识虽然学过但是已经忘了。

（2）大部分学生家里有计算机，但是主要用于娱乐，用来学习的不多。

（3）对 office 办公软件 Word、Excel、Powerpoint、Frontpage 及 Inernet 应用的掌握情况, 学生对于各 种上网操作如收发邮件、搜索资料、下载安装软件等掌握情况最好, 其次是 Word 操作, Excel、Powerpoint 操作只有三分之一的同学会做, Frontpage 只有四分之一的同学会部分操作。

(4) 对计算机的理论知识, 如信息安全、计算机网络等, 学生普遍了解的不多。

（5）只有少部分学生在中学时接触过计算机编程语言, 只是一般了解。

（6）大约一半的新生计算机基础较差, 大约 40\%的学生有一定的计算机操作能力, 有 $10 \%$ 的新生有 较高的计算机应用能力。

\section{5 分级教学建议}

根据以上统计分析结果和因材施教的教育理念, 建议根据新生的计算机知识掌握情况进行分级教学。 具体实施步骤如下:

(1) 在新生入学后, 首先印发分级教学的具体实施方案, 做好分级教学的宣传工作, 动员新生积极 参与。

（2）然后利用下午自习时间分批对全校新生进行计算机基础摸底考试, 试题难度可比平时考试稍低, 采用在机房下发电子试题, 学生在线答题并提交的方式。

(3) 根据考试成绩将学生分成多个 A 班和 B 班, 其中 A 班是成绩较好, 具有一定计算机基础的学生, $\mathrm{B}$ 班是成绩较差, 计算机基础较弱或没接触过计算机的学生。

科学分级是分级教学的第一步, 然后就要严格按照教学大纲的要求, 合理安排各级别学生的教学内容。 对 $\mathrm{A}$ 班学生来说, 有着较高的计算机操作能力, 可以为他们选用高级别的教材, 针对学生的专业特点 补充有针对性的技能操作项目, 还可以开设其他计算机选修课供学生选择学习。在教学过程中要注意学生 创新能力的培养, 而不仅仅是传授知识和技能。如举办设计大赛或各种竞赛, 由教师出题, 学生自己动手 设计有创造性的作品, 激发学生的创造性思维, 优秀的作品还可以在校内公开展览, 这样既能营造一种良 好的学习氛围, 又能充分调动学生的积极性和主动性。 
对于基础较差的 B 班学生, 可以为他们选用通俗易懂的入门教材, 教学时注意学生的理解领会情况, 必要时可以减慢教学进度, 以学生听懂会操作为主要目标, 课下可提供更多的时间让学生练习, 树立学生 的自信心, 提高学习兴趣。当他们的学习达到一定程度后, 鼓励他们向 $\mathrm{A}$ 班学生靠拢。 $\mathrm{B}$ 班的学生通过努 力, 有可能赶上 A 班学生, 甚至更加出色。

\section{6 结语}

分级教学的目的是全面提高大学生的计算机知识水平和应用能力, 同时有利于授课教师开展教学, 具 体实施要有更高的要求。相信我们本着因材施教的原则, 按教学规律开展教学, 不断完善分级教学方案, 就能更高质量完成教学任务。

\section{参考文献:}

[1] 期刊——杨俏炜. 《分层教学法在计算机基础课程教学中的应用》 [J]. 电脑知识与技术, 2016 年, 第 10 期: 164-165

[2] 期刊一一李昕. 《分层教学在计算机基础课程中的应用研究》 $[\mathrm{J}]$. 辽宁工业大学学报（社会科学版）, 2013 年, 第 15 卷第 3 期: $129-131$

[3] 期刊——李文娟. 《分层教学在计算机应用基础课程中的应用》 [J]. 学园, 2015 年, 03 期： 78

[4] 期刊一一钟燕. 《分层教学在中职计算机应用基础课程教学中的应用研究》 [J]. 广西教育, 2014 年, 第 14 期: $20-21$

[5] 期刊一一胡灿. 《分层教学与项目化相结合的《计算机应用基础》课改模式》 [J]. 通讯世界, 2015 年, 第 24 期: 275

[6] 期刊一一蒋凌云; 周显龙. 《《计算机应用基础》课程分层教学的设计与实践》 $[\mathrm{J}]$. 贵州广播电视大学学报, 2012 年，第 2 期: 9-11

[7] 期刊一一间利霞. 《浅谈高职院校计算机应用基础课程教学改革》 [J]. 电子世界, 2016 年, 第 23 期: $16+18$

[8] 期刊——姚江梅. 《计算机基础分层教学模式在高职院校中的探讨》 [J]. 工程技术研究, 2016 年, 第 8 期: $229-231$

[9] 期刊一一王兆华. 《高校计算机基础课程分层教学之分层方法探讨》 $[\mathrm{J}]$. 计算机教育, 2011 年, 第 8 期: 99-102

[10] 期刊一一李妮; 王琴竹. 《计算机基础课程实验中的模块化分层教学》 $[\mathrm{J}]$. 运城学院学报, 2013 年, 第 5 期: $74-76$

\section{References:}

[1] Journal-Yang Qiaowei. Application of Hierarchical Approach in Teaching of Computer Basic Courses [J].Computer Knowledge and Technology, 2016 (Volume 10):164-165

[2] Journal-Li Xin. Applied research on Group Teaching in Computer Basic Courses [J].Journal of Liaoning Institute of Technology (Social Science Edition), 2013 (Volume 10-3):129-131

[3] Journal-Li Wenjuan. Application of Hierarchical Approach in Teaching of Computer Basic Courses [J].Learing Garden, 2015 (3):78

[4] Journal-Zhong Yan. Application Research on Layered Teaching in Basic Courses Teaching of Computer Application in Secondary Vocational Schools [J].Guangxi Education,2014(14): 20-21 
[5] Journal-Hu Can. Curriculum Reform Models of Basic Computer Applications by combining Layered Teaching and Projectization [J].Telecom World, 2015(24):275

[6] Journal-Jiang Lingyun, Zhou Xianlong. Design and Practice of Layered Teaching in Basic Computer Application [J].Journal of Guizhou Radio \& Television University, 2012(2):9-11

[7] Journal-Yan Lixia. On Teaching Reform of Basic Computer Application Courses in Higher Vocational Colleges [J].Electronics World, 2016(23):16+18

[8] Journal-Yao Jiangmei. Research on Basic Computer Layer Teaching Mode in Higher Vocational Colleges [J].Engineering and Technological Research, 2016(8):229-231

[9] Journal-Wang Zhaohua. Discussion on Hierarchical of Group Teaching in Basic Computer Course of College [J].Computer Education, 2011(8):99-102

[10]Journal-Li Ni, Wang Qinzhu. Modular Hierarchical in Computer Basic Courses Experimental Teaching [J].Journal of Yuncheng University, 2013(5):74-76 